\title{
Cordyceps sinensis oral liquid prolongs the lifespan of the fruit fly, Drosophila melanogaster, by inhibiting oxidative stress
}

\author{
YINGXIN ZOU $^{1 *}$, YUXIANG LIU $^{1,2^{*}}$, MINGHUA RUAN $^{1}$, XU FENG $^{1}$, \\ JIACHUN WANG ${ }^{1}$, ZHIYONG CHU $^{1}$ and ZESHENG ZHANG ${ }^{2}$ \\ ${ }^{1}$ Naval Medical Research Institute, Shanghai 200433; ${ }^{2}$ Department of Food Engineering and Biotechnology, \\ Tianjin University of Science and Technology, Tianjin 300457, P.R. China
}

Received January 23, 2015; Accepted July 16, 2015

DOI: $10.3892 / \mathrm{ijmm} .2015 .2296$

\begin{abstract}
This study investigated the effect of Cordyceps sinensis oral liquid (CSOL) on the lifespan of Drosophila melanogaster (fruit fly). Following the lifelong treatment of fruit flies with CSOL, lifespan was examined. The activity of copper-zinc-containing superoxide dismutase 1 (SOD1), manganese-containing superoxide dismutase 2 (SOD2) and catalase (CAT), as well as the lipofuscin (LF) content were determined. The mRNA levels of SOD1, SOD2 and CAT were quantified by qPCR. Hydrogen peroxide $\left(\mathrm{H}_{2} \mathrm{O}_{2}\right)$ and paraquat were used to mimic the effects of damage caused by acute oxidative stress. D-galactose was used to mimic chronic pathological aging. CSOL significantly prolonged the lifespan of the fruit flies under physiological conditions. The activity of SOD1 and CAT was upregulated, and LF accumulation was inhibited by CSOL. CSOL had no effect on the transcriptional levels (mRNA) of these enzymes. The survival time of the fruit flies which were negatively affected by exposure to $\mathrm{H}_{2} \mathrm{O}_{2}$ or paraquat was significantly prolonged by CSOL. In fruit flies pathologically aged by epxosure to D-galactose, CSOL also significantly prolonged their lifespan, upregulated the activity of SOD1 and CAT, and inhibited LF accumulation. The findings of our study indicate that CSOL prolongs the lifespan of fruit flies through an anti-oxidative stress pathway involving the upregulation of SOD1 and CAT activity and the inhibition of LF accumulation. CSOL may thus be explored as a novel agent for slowing the human aging process.
\end{abstract}

Correspondence to: Professor Zhiyong Chu, Naval Medical Research Institute, 880 Xiangyin Road, Shanghai 200433, P.R. China E-mail: zhiyongchuleader@163.com

Professor Zesheng Zhang, Department of Food Engineering and Biotechnology, Tianjin University of Science and Technology, 29 Thirteenth Avenue, Tianjin Economic-Technological Development Area, Tianjin 300457, P.R. China

E-mail: zhangzesheng@tust.edu.cn

${ }^{*}$ Contributed equally

Key words: Cordyceps sinensis, aging, lifespan, oxidative stress, Drosophila melanogaster

\section{Introduction}

Increased rates of population aging are causing the problems associated with aging to attract increasing attention. By the year 2050, the number of individuals over the age of 80 will triple globally (1). More than $70 \%$ of individuals over 65 years of age suffer from at least two chronic diseases, such as heart disease, stroke, cancer, arthritis and diabetes (2). Studies of food or medicine as a means of extending longevity have been taking place since ancient times.

The underlying mechanisms of aging remain unknown, despite tremendous progress being made in this area. There is growing evidence to suggest that oxidative stress increases with age (3). The endogenous enzymes, superoxide dismutase (SOD) and catalase (CAT), are involved in the human antioxidant defense network of human (4). Lipofuscin (LF) accumulation, which is deemed to be a hallmark of aging (5), has been shown to be related to the loss of protein homeostasis (6) and the rate of accumulation has been linked to age-dependent mortality (7).

Recently, great interest has arisen in the possibility that antioxidants, particularly naturally occurring antioxidants from edible materials, may reduce the risk of aging (8).

Cordyceps sinensis, one of the most valued edible, medical entomopathogenic fungi, used in traditional Chinese medicine for thousands of years, is commonly used as a tonic for promoting vitality and longevity, as well as a herbal medicine for treating various intractable diseases $(9,10)$. It has been demonstrated to possess multiple pharmacological properties, such as antioxidant (11), immunomodulatory (12) and antitumor (13) properties. Furthermore, Cordyceps sinensis extract has been reported to improve learning and memory function in a mouse model of D-galactose-induced aging (14). The aqueous polysaccharides of Cordyceps taii have been shown to possess antioxidant activity in a mouse model of D-galactose-induced aging (15). However, to the best of our knowlewdge, the antiaging effects of Cordyceps sinensis in vivo, under physiological conditions, have not been confirmed to date.

Cordyceps sinensis oral liquid (CSOL) was studied and manufactured by the Naval Medical Research Institute (Shanghai, China) (16), and has been used as an immunomodulator, an adjunctive therapy during chemoradiation treatment and to ameliorate chronic bronchitis more than twenty years (17). In a previous study of ours, we demonstrated that CSOL inhibited 
damage induced by oxygen and glucose deprivation in vitro (unpublished data) and thus, CSOL has potential for use as an agent to ameliorate stroke-induced brain damage.

The aim of the present study was to assess the anti-aging/ life-prolonging effects of CSOL in Drosophila melanogaster (D. melanogaster, fruit fly) under conditions of physiological (normal) and pathological (premature) aging. In addition, the mechanisms involving the anti-oxidative stress pathway were investigated.

\section{Materials and methods}

CSOL. CSOL (lot no. 20130906; the Naval Medical Research Institute), consists of wild Cordyceps sinensis extract (CSE) and Cordyceps sinensis mycelial fermentation broth (CSMFB). CSE and CSMFB were mixed in a ratio of $6: 4(\mathrm{v} / \mathrm{v})$. The CSOL concentration was expressed as $100 \mathrm{mg}$ of wild Cordyceps sinensis per $10 \mathrm{ml}$ of CSOL $(100 \mathrm{mg} / 10 \mathrm{ml})$. Adenosine (no less than $1.0 \mathrm{mg} / 100 \mathrm{ml}$ ) was used as a marker for CSOL quality control and was quantified using an Agilent 1260 HPLC apparatus (Agilent Technologies, Santa Clara, CA, USA). This sample of CSOL was found to have an adenosine concentration of $3.94 \mathrm{mg} / 100 \mathrm{ml}$ (Fig. 1). In the present study, CSOL was pre-filtered by sterile membrane filtration under aseptic conditions and stored at $4^{\circ} \mathrm{C}$ for use.

Fruit fly strain and culture conditions. The wild-type OregonK-strain of the D. melanogaster fruit fly was kindly provided by Professor Zesheng Zhang of the Tianjin University of Science and Technology, Tianjin, China. The fruit flies were housed in $50 \mathrm{ml}$ plastic vials containing $5 \mathrm{ml}$ of culture medium, and the vials were kept at $25 \pm 1^{\circ} \mathrm{C}, 60 \pm 5 \%$ humidity on a 12:12 h light/dark cycle. The basal culture medium consisted of $72 \mathrm{~g}$ cornmeal, $72 \mathrm{~g}$ glucose (63005518), $10 \mathrm{~g}$ yeast (Angel Yeast Co., Ltd., Yichang, China), 6 g agar (10000582), $40 \mathrm{ml}$ antiseptic (1\% ethyl 4-hydroxybenzoate in $75 \%$ alcohol) (Sigma-Aldrich, St. Louis, MO, USA) and water to prepare $500 \mathrm{ml}$ of medium. Glucose and agar were purchased from Sinopharm Chemical Regent Co., Ltd. (Shanghai, China).

The mixture was cooked and poured into vials $(5 \mathrm{ml}$ in each). All relative protocols were approved by the Local Institutional Committee of Naval Medical Research Institute.

Determination of the effects of CSOL on fruit flies under physiological conditions

Lifespan assay. A total of 800 male fruit flies (eclosion within 8 h) were randomly divided into 4 groups as follows: the control group, and the groups treated with $0.02,0.06$ and $0.20 \mathrm{mg} / \mathrm{ml}$ CSOL. The fruit flies in the CSOL-treated groups were fed with culture medium supplemented with CSOL at final concentrations of $0.02,0.06$ and $0.20 \mathrm{mg} / \mathrm{ml}$, respectively. The fruit flies in the control group were fed with basal culture medium. The fruit flies were transferred to fresh culture medium twice a week. The number of dead fruit flies was recorded every 3 days until all flies died. The survival time was observed. The lifespan curve was drawn, and the median and mean lifespans were calculated. Maximum lifespan was calculated as the average lifespan of the longest surviving $10 \%$ of the fruit fly population.

Enzyme assays. To examine the effects of CSOL on antioxidant enzymes, male fruit flies (eclosion within $8 \mathrm{~h}$ ) were randomly divided into 2 groups: the control group and the $0.06 \mathrm{mg} / \mathrm{ml}$ CSOL-treated group. The daily treatment was as described above. On days 0,25 and 45, the fruit flies were collected for the determination of the activity of SOD and CAT, and the LF content. Copper-zinc-containing SOD (SOD1) activity, manganese-containing SOD (SOD2) activity and CAT activity were detected according to the manufacturer's instructions. The SOD activity assay kit (S0103) was purchased from Beyotime Biotechnology Co., Ltd. (Haimen, China) and the CAT activity assay kit (A007-1) was purchased from Nanjing Jiancheng Bioengineering Institute (Nanjing, China). The LF concentration was determined as previously described (18). Briefly, the fruit fly homogenate was extracted with chloroform:methanol $(2: 1, \mathrm{v} / \mathrm{v})$ and centrifuged at $3000 \mathrm{x} \mathrm{g}$ for $10 \mathrm{~min}$. Chloroform (40064966) and methanol (40064292) were purchased from Sinopharm Chemical Regent Co., Ltd. The absorbance (excitation, $360 \mathrm{~nm}$ and emission, $430 \mathrm{~nm}$ ) was measured using a Fluorospectrophotometer-850 (Hitachi, Tokyo, Japan). Standardization was carried out with a freshly prepared solution of quinine sulfate. Quinine sulfate (LQ3281) was purchased from Hefei Bomei Biotechnology Co., Ltd. (Hefei, China). The LF concentration was calculated from a calibration curve of quinine sulfate and expressed as $\mu \mathrm{g} / \mathrm{mg}$ body weight.

Measurement of the transcriptional levels of antioxidant enzymes. This experiment was designed to examine the effect of CSOL treatment on the mRNA levels of antioxidant genes in fruit flies. Male fruit flies (eclosion within $8 \mathrm{~h}$ ) were randomly divided into 2 groups: the control and $0.06 \mathrm{mg} / \mathrm{ml}$ CSOL-treated group, and housed as described above. The fruit flies were collected on days $0,15,25,35,45$ and 55 . The mRNA levels of SOD1, SOD2 and CAT were quantified by quantitative PCR (qPCR) as described in our previous study (19). Briefly, total RNA was extracted using Trizol reagent (Invitrogen, Carlsbad, CA, USA), $2 \mu \mathrm{g}$ RNA was reverse transcribed into cDNA using RNA reverse transcriptase (Promega, Madison, WI, USA), and qPCR was performed using an Option Monitor 3 Real-Time PCR System (Bio-Rad, Hercules, CA, USA) on an ABI 7500 PCR instrument (Applied Biosystems, Carlsbad, CA, USA) with SYBR Premix Ex Taq ${ }^{\mathrm{TM}}$ Mixture (Takara, Otsu, Japan) following the manufacturer's instructions. The primers used were as follows: SOD1 sense, 5'-CTGCT CTGCTACGGTCACAC-3' and antisense, 5'-ACAGCTTT AACCACCATTTCG-3'; SOD2 sense, 5'-CCACATCAACC ACACCATCT-3' and antisense, 5'-CAGTTTGCCCGAC TTCTTGT-3'; CAT sense, 5'-TTCGATGTCACCAAGG TCTG-3' and antisense, 5'-TGCTCCACCTCAGCAAAGTA-3'; and RP49 sense, 5'-ACTTCATCCGCCACCAGTC-3' and antisense, 5'-ATCT CGCCGCAGTAAACG-3'. RP49 was used as an internal control. The results were analyzed using the $2^{-\Delta \Delta \mathrm{Ct}}$ method.

Determination of the effects of CSOL on fruit flies exposed to acute oxidative stress

Survival time [hydrogen peroxide $\left(\mathrm{H}_{2} \mathrm{O}_{2}\right)$ exposure] assay. Oxygen-containing free radicals are considered to be involved in the mechanisms of aging (20). $\mathrm{H}_{2} \mathrm{O}_{2}$ is usually used to mimic damage induced by oxidative stress (21). The present experiment was designed to examine the protective effects of CSOL against acute oxidative stress induced by $\mathrm{H}_{2} \mathrm{O}_{2}$ in fruit flies. $\mathrm{H}_{2} \mathrm{O}_{2}$ (lot: 20120601) was purchased from Sinopharm 

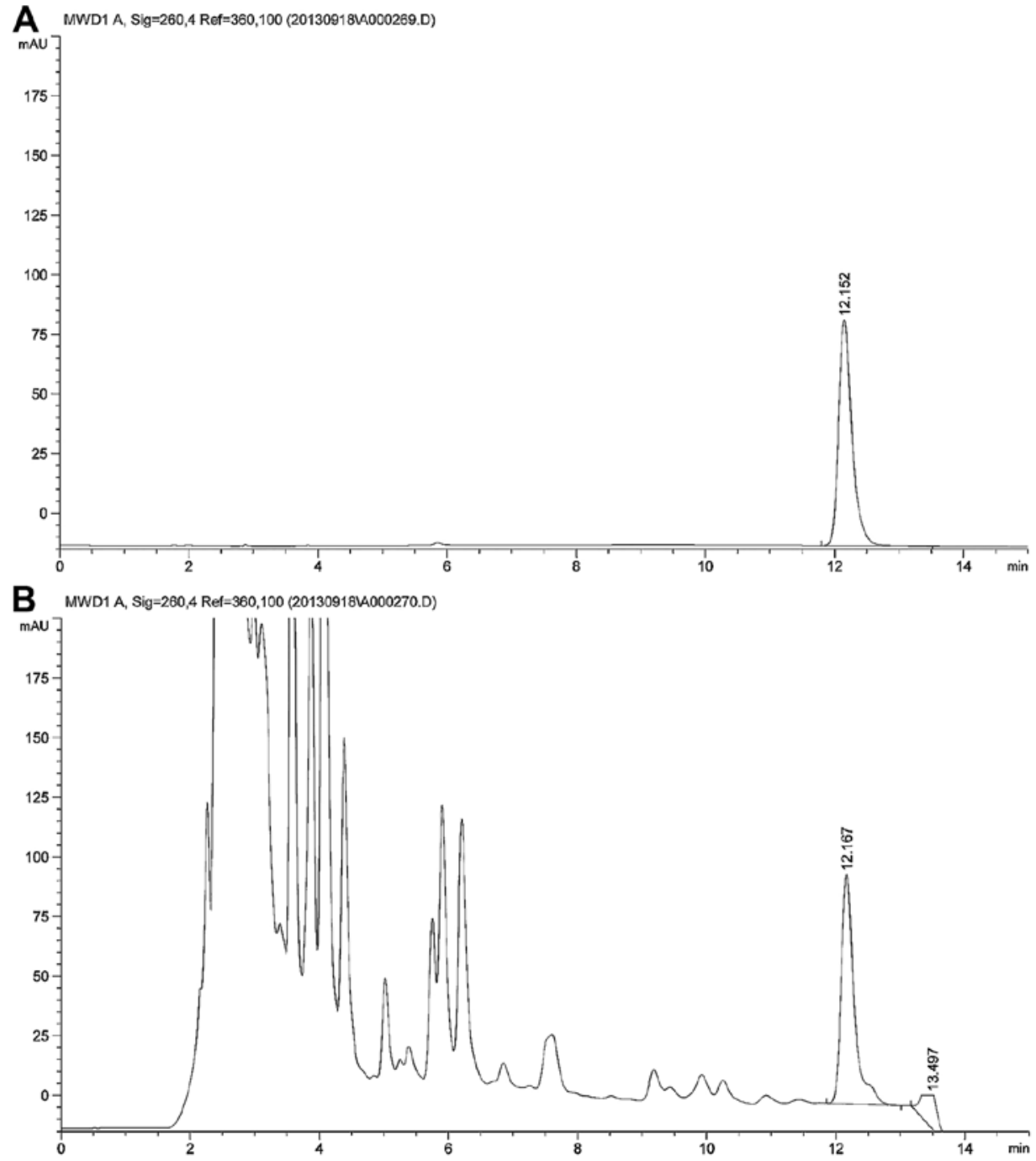

Figure 1. HPLC chromatograms of adenosine levels in Cordyceps sinensis oral liquid (CSOL) (20130906). This sample of CSOL was found to have an adenosine concentration of $3.94 \mathrm{mg} / 100 \mathrm{ml}$. (A) HPLC chromatogram of adenosine standard. (B) HPLC chromatogram of CSOL.

Chemical Regent Co., Ltd. Male fruit flies (eclosion within $8 \mathrm{~h}$ ) were divided into groups and housed as described above. On day 25 , the fruit flies were first starved for $2 \mathrm{~h}$, and then transferred into new vials containing a filter paper saturated with $1 \mathrm{ml}$ of $30 \% \mathrm{H}_{2} \mathrm{O}_{2}$. The numbers of dead fruit flies was recorded every $4 \mathrm{~h}$ until all flies died.

Survival time (paraquat exposure) assay. Paraquat (1,1'-dimethyl-4,4'-bipyridinium dichloride; Sigma-Aldrich) has been reported to induce mitochondrial dysfunction and increase reactive oxygen species (ROS) production (22). The present experiment was designed to examine the protective effects of CSOL against acute oxidative stress induced by paraquat in fruit flies. Male fruit flies (eclosion within $8 \mathrm{~h}$ ) were grouped and housed as described above. On day 25, the fruit flies were first starved for $2 \mathrm{~h}$, and then transferred into new vials containing a filter paper saturated with $1 \mathrm{ml}$ of $20 \mathrm{mM}$ paraquat in a $6 \%$ glucose solution. The number of dead fruit flies was recorded every $4 \mathrm{~h}$ until all flies died.

Determination of the effects of CSOL on fruit flies under conditions of pathological aging induced by D-galactose.
The chronic administration of D-galactose has been reported to accelerate aging (23) and is therefore, regarded as suitable for use in models of aging. The excessive intake of D-galactose may contribute to ROS generation through the oxidative metabolism of D-galactose and through the increased formation of advanced glycation end products (24). This experiment was carried out to examine the effects of CSOL on a fruit fly model of D-galactose-induced aging. D-galactose (SG075003) was purchased from Sigma-Aldrich.

Lifespan assay. Male fruit flies (eclosion within $8 \mathrm{~h}$ ) were randomly divided into 5 groups as follows: the control group, the model group, and the groups treated with $0.02,0.06$ and $0.20 \mathrm{mg} / \mathrm{ml} \mathrm{CSOL}$. The fruit flies in the model group and the CSOL groups were exposed to culture medium containing $6.5 \% \mathrm{D}$-galactose for the duration of the experiment. The culture medium of the fruit flies in the CSOL-treated groups was supplemented with CSOL at a final concentration of 0.02 , 0.06 and $0.20 \mathrm{mg} / \mathrm{ml}$. The fruit flies in the control group were fed with basal culture medium. The daily treatment was the same as that described above. The lifespan curve, the median, mean and maximum lifespans were determined as described above. 
Table I. Lifespan parameters in fruit flies following lifelong treatment with CSOL.

\begin{tabular}{lccccc}
\hline $\begin{array}{l}\text { Group } \\
(\mathrm{mg} / \mathrm{ml})\end{array}$ & $\begin{array}{c}\text { Median lifespan } \\
\text { (days) }\end{array}$ & $\begin{array}{c}\text { Mean lifespan } \\
\text { (days) }\end{array}$ & $\begin{array}{c}\text { Change from } \\
\text { control }(\%)\end{array}$ & Log-rank (vs. control) & $\begin{array}{c}\text { Maximum lifespan } \\
\text { (days) }\end{array}$ \\
\hline Control & $38.0 \pm 1.0$ & $35.3 \pm 0.8$ & - & - & $57.8 \pm 5.8$ \\
0.02 & $45.0 \pm 1.5$ & $44.2 \pm 1.0$ & 25 & $\chi^{2}=53.167, \mathrm{P}<0.001$ & $67.3 \pm 3.5^{\mathrm{a}}$ \\
0.06 & $47.0 \pm 1.1$ & $46.1 \pm 1.0$ & 31 & $\chi^{2}=79.677, \mathrm{P}<0.001$ & $69.0 \pm 3.4^{\mathrm{a}}$ \\
0.20 & $49.0 \pm 0.8$ & $46.6 \pm 0.9$ & 32 & $\chi^{2}=87.063, \mathrm{P}<0.001$ & $66.7 \pm 5.0^{\mathrm{a}}$ \\
\hline
\end{tabular}

Maximum lifespan was calculated as the average lifespan of the longest surviving $10 \%$ of fruit flies in each group ( $n=200$; ${ }^{\text {a }}<0.001$ vs. the control group). CSOL, Cordyceps sinensis oral liquid.

Enzyme assays. The effects of CSOL on pathological aging induced by D-galactose were further evaluated, and thus, the activity of SOD1, SOD2 and CAT, and LF accumulation were detected. Male fruit flies (eclosion within $8 \mathrm{~h}$ ) were divided into groups and housed as described above. On day 25 , the fruit flies were collected to detect the enzyme activity. The detection methods were as described above.

Statistical analysis. Data are expressed as the means \pm SD. Comparisons among groups were made by analysis of variance (ANOVA) followed by Dunnett's t-test. The Log-rank test was used to evaluate the equality of the survival curves. P-values $<0.05$ were considered to indicate statistically significant differences.

\section{Results}

Effects of CSOL on fruit flies under physiological conditions Lifelong treatment with CSOL prolongs the lifespan of fruit flies. Lifelong treatment with CSOL at concentrations of 0.02 , 0.06 and $0.20 \mathrm{mg} / \mathrm{ml}$ significantly prolonged the lifespan of the fruit flies (Fig. 2). The median and mean lifespan parameters shown in Table I confirmed these results. Treatment with CSOL at $0.02,0.06$ and $0.20 \mathrm{mg} / \mathrm{ml}$ prolonged the mean lifespan of the fruit flies (vs. the control) by 25,31 and $32 \%$, respectively (Table I).

CSOL enhances the activity of most antioxidant enzymes. To eluciate the mechanisms responsible for the lifespanprolonging effect of CSOL on fruit flies, we examined the effects of CSOL on the activity of the antioxidant enzymes, SOD1, SOD2 and CAT. Compared with the control group at the same time point, treatment with CSOL significantly enhanced the activity of SOD1 (Fig. 3A) and CAT (Fig. 3C), and inhibited LF accumulation (Fig. 3D). CSOL had no significant effect on the activity of SOD2, although its activity was found to significantly decrease with age (Fig. 3B).

CSOL has no effect on the transcriptional levels of antioxidant enzymes. To determine the effects of CSOL on the transcriptional levels of antioxidant enzymes, we measured the mRNA levels of SOD1, SOD2 and CAT in the fruit flies. Treatment with CSOL was found to have no effect on the mRNA levels of SOD1 (Fig. 4A), SOD2 (Fig. 4B) and CAT (Fig. 4C). In the control group, the mRNA level of SOD2 was highest at eclosion, and then decreased to a relative low level from day 15 (Fig. 4B).

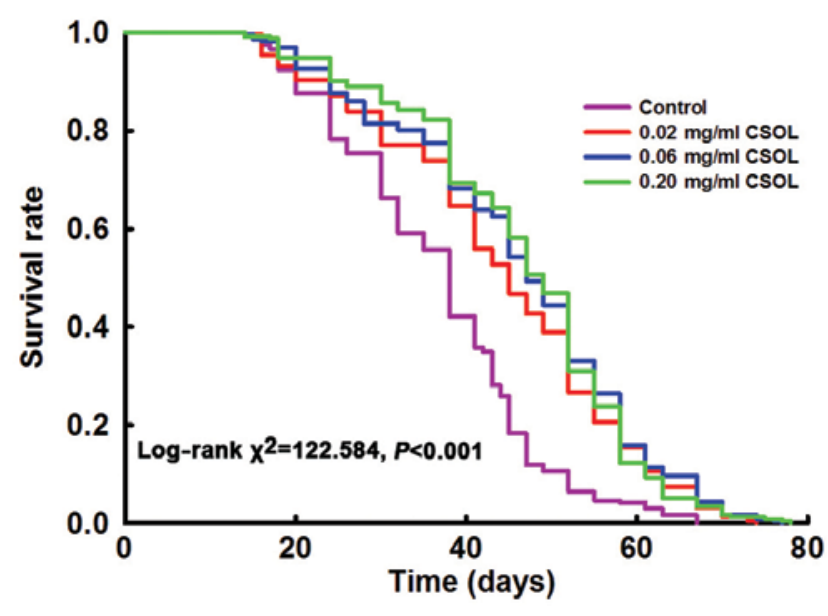

Figure 2. Lifelong treatment with Cordyceps sinensis oral liquid (CSOL) prolongs the lifespan of fruit flies $(\mathrm{n}=200)$. Fruit flies received lifelong treatment with CSOL at concentrations of $0.02,0.06$ and $0.20 \mathrm{mg} / \mathrm{ml}$. Fruit flies in the control group were fed with basal culture medium. The number of dead flies was recorded every 3 days until all flies died. Data were analyzed by the log-rank test.

CSOL prolongs the survival time of fruit flies subjected to $\mathrm{H}_{2} \mathrm{O}_{2}$ - or paraquat-induced oxidative stress

Exposure to $\mathrm{H}_{2} \mathrm{O}_{2}$. The results revealed that treatment with CSOL significantly prolonged the survival time of the fruit flies exposed to $\mathrm{H}_{2} \mathrm{O}_{2}$ (Fig. 5A). Treatment with CSOL at concentrations of 0.06 and $0.20 \mathrm{mg} / \mathrm{ml}$ significantly prolonged the mean survival time by 13 and $16 \%$, respectively, vs. the model group (Table II). Treatment with CSOL at a concentration of $0.02 \mathrm{mg} / \mathrm{ml}$ also prolonged the mean survival time of the fruit flies exposed to $\mathrm{H}_{2} \mathrm{O}_{2}$; however, no statistically significant difference was observed. These results suggest an obvious dose-effect relationship.

Exposure to paraquat. The results revealed that treatment with CSOL significantly prolonged the survival time of the fruit flies exposed to paraquat (Fig. 5B). Treatment with CSOL at concentrations of 0.06 and $0.20 \mathrm{mg} / \mathrm{ml}$, significantly prolonged the mean survival time by 24 and $15 \%$, respectively, vs. the model group (Table II). Treatment with CSOL at a concentration of $0.02 \mathrm{mg} / \mathrm{ml}$ prolonged the mean survival time of the fruit flies exposed to paraquat; however, no statistically significant difference was observed. 
A
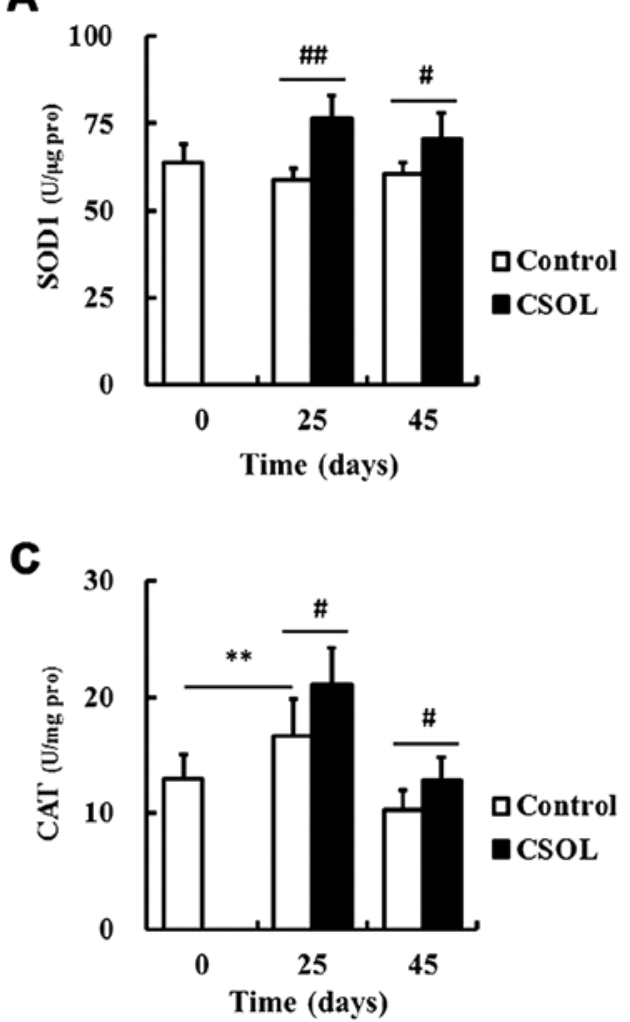
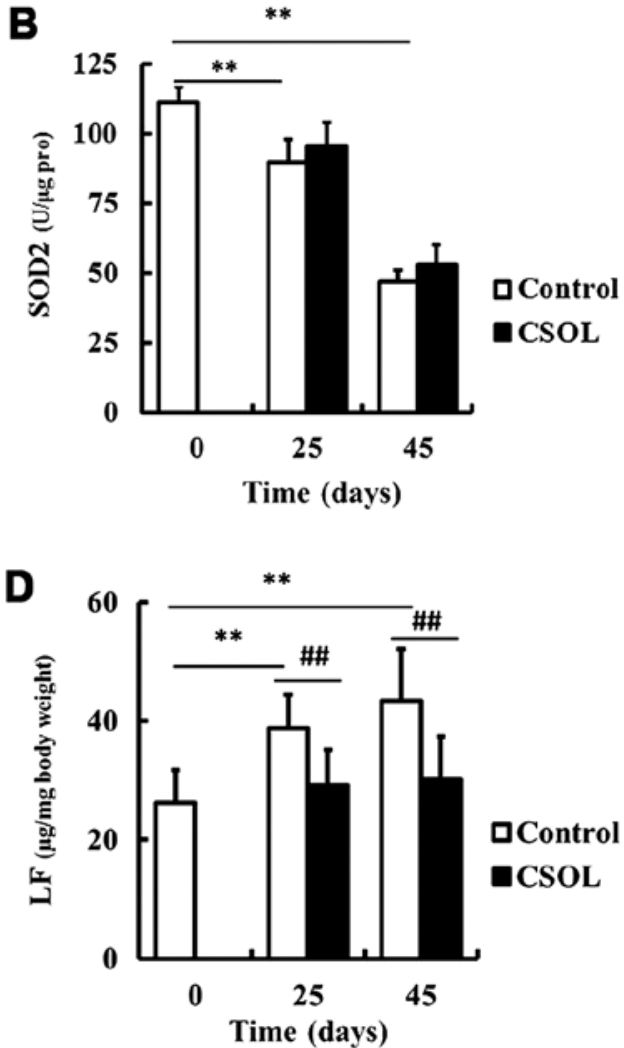

Figure 3. Effect of Cordyceps sinensis oral liquid (CSOL) on antioxidant enzyme activity in fruit flies. Fruit flies (eclosion within 8 h) were treated with culture medium containing $0.06 \mathrm{mg} / \mathrm{ml}$ of CSOL. Fruit flies in the control group were fed with basal culture medium. On days 0,25 and 45 , fruit flies were collected for the detection of the activity of the following antioxidant enzymes. Data are presented as the means \pm SD. Data were analyzed by ANOVA followed by Dunnett's t-test. $\mathrm{n}=6,{ }^{* *} \mathrm{P}<0.01$ vs. day $0 ;{ }^{*} \mathrm{P}<0.05$ and ${ }^{\# \#} \mathrm{P}<0.01$ vs. the control group at the same time point. (A) Treatment with CSOL enhanced the activity of superoxide dismutase (SOD)1 in fruit flies on day 25. (B) Treatment with CSOL had no effect on the activity of SOD2 in fruit flies. (C) Treatment with CSOL enhanced the activity of catalase (CAT) in fruit flies on days 25 and 45. (D) Lipofuscin (LF) accumulation increased with age, and treatment with CSOL significantly inhibited this accumulation on days 25 and 45 .
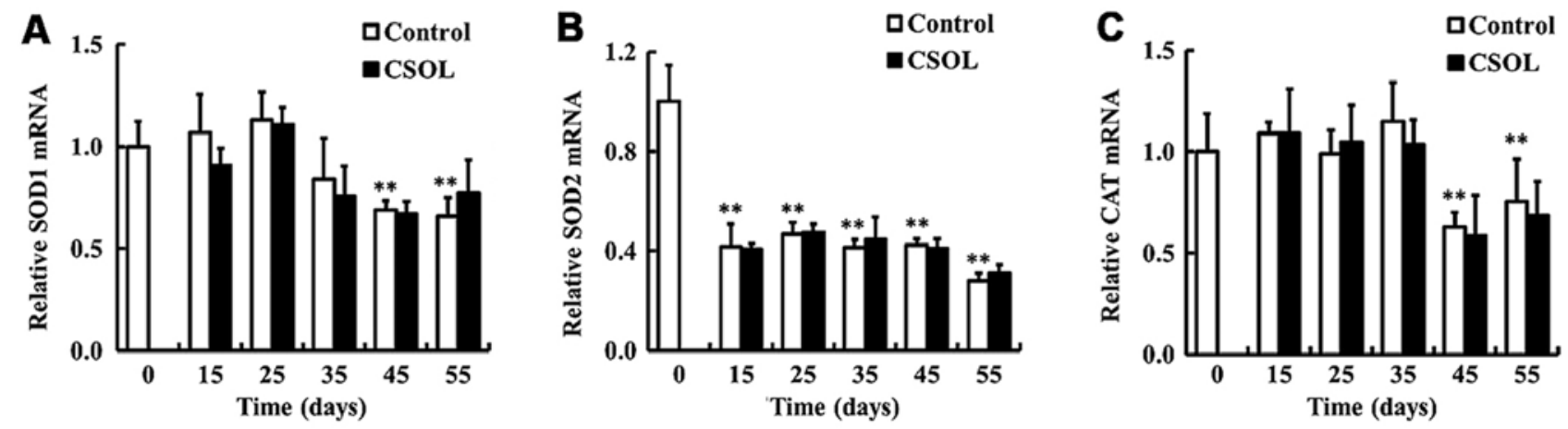

Figure 4. Treatment with Cordyceps sinensis oral liquid (CSOL) has no effect on the mRNA levels of antioxidant genes in fruit flies. Fruit flies (eclosion within $8 \mathrm{~h}$ ) were treated with culture medium containing $0.06 \mathrm{mg} / \mathrm{ml} \mathrm{CSOL}$. Fruit flies in the control group were fed with basal culture medium. On days $0,15,25,35$, 45 and 55, fruit flies were collected for the detection of the mRNA levels of antioxidant genes. (A) Relative superoxide dismutase (SOD)1 mRNA. (B) Relative SOD2 mRNA. (C) Relative catalase (CAT) mRNA. Data are presented as the means \pm SD. Data were analyzed by ANOVA. $n=6,{ }^{* *} \mathrm{P}<0.01$.

CSOL prolongs the lifespan and inhibits oxidative stress in patholgically-aged fruit flies (induced by $D$-galactose). The addition of D-galactose to the culture medium significantly shortened the maximum lifespan of the fruit flies from $72.2 \pm 5.7$ days in the control group to $57.7 \pm 2.6$ days in the model group (Fig. 6A and Table III). However, treatment with CSOL at concentrations of $0.02,0.06$ and $0.20 \mathrm{mg} / \mathrm{ml}$, modestly inhibited the lifespan shortening effect by 6,8 and
$12 \%$, respectively (Table III). These results were supported by the parameters of the mean and maximum lifespans (Table III).

Since we found that CSOL enhanced the activity of SOD1 and CAT, inhibited LF accumulation, and had no effect on the activity of SOD2, we measured the levels of these enzymes in fruit flies pathologically-aged by D-galactose in order to further confirm the effect of CSOL on oxidative stress under these conditions. SOD1 (Fig. 6B) and CAT (Fig. 6D) activity 
Table II. Survival parameters in fruit flies exposed to $\mathrm{H}_{2} \mathrm{O}_{2}$ or paraquat and treated with CSOL.

\begin{tabular}{|c|c|c|c|c|}
\hline $\begin{array}{l}\text { Group } \\
(\mathrm{mg} / \mathrm{ml})\end{array}$ & $\begin{array}{l}\text { Median survival } \\
\text { time }(\mathrm{h})\end{array}$ & $\begin{array}{l}\text { Mean survival } \\
\text { time (h) }\end{array}$ & $\begin{array}{l}\text { Change from } \\
\text { model }(\%)\end{array}$ & $\begin{array}{l}\text { Log-rank } \\
\text { (vs. model) }\end{array}$ \\
\hline \multicolumn{5}{|l|}{$\mathrm{H}_{2} \mathrm{O}_{2}$} \\
\hline Model & $20.0 \pm 0.5$ & $18.9 \pm 0.5$ & - & - \\
\hline 0.02 & $20.0 \pm 0.7$ & $19.5 \pm 0.5$ & 3 & $\chi^{2}=1.107, \mathrm{P}=0.293$ \\
\hline 0.06 & $20.0 \pm 0.6$ & $21.3 \pm 0.5$ & 13 & $\chi^{2}=11.170, P<0.001$ \\
\hline 0.20 & $20.0 \pm 0.5$ & $22.0 \pm 0.5$ & 16 & $\chi^{2}=21.605, P<0.001$ \\
\hline \multicolumn{5}{|l|}{ Paraquat } \\
\hline Model & $20.0 \pm 0.8$ & $21.7 \pm 0.6$ & - & - \\
\hline 0.02 & $20.0 \pm 0.9$ & $22.3 \pm 0.6$ & 3 & $\chi^{2}=0.756, P=0.385$ \\
\hline 0.06 & $28.0 \pm 0.7$ & $26.8 \pm 0.6$ & 24 & $\chi^{2}=27.739, P<0.001$ \\
\hline 0.20 & $28.0 \pm 0.9$ & $25.0 \pm 0.7$ & 15 & $\chi^{2}=13.091, \mathrm{P}<0.001$ \\
\hline
\end{tabular}

CSOL, Cordyceps sinensis oral liquid. Fruit flies $(\mathrm{n}=200)$.
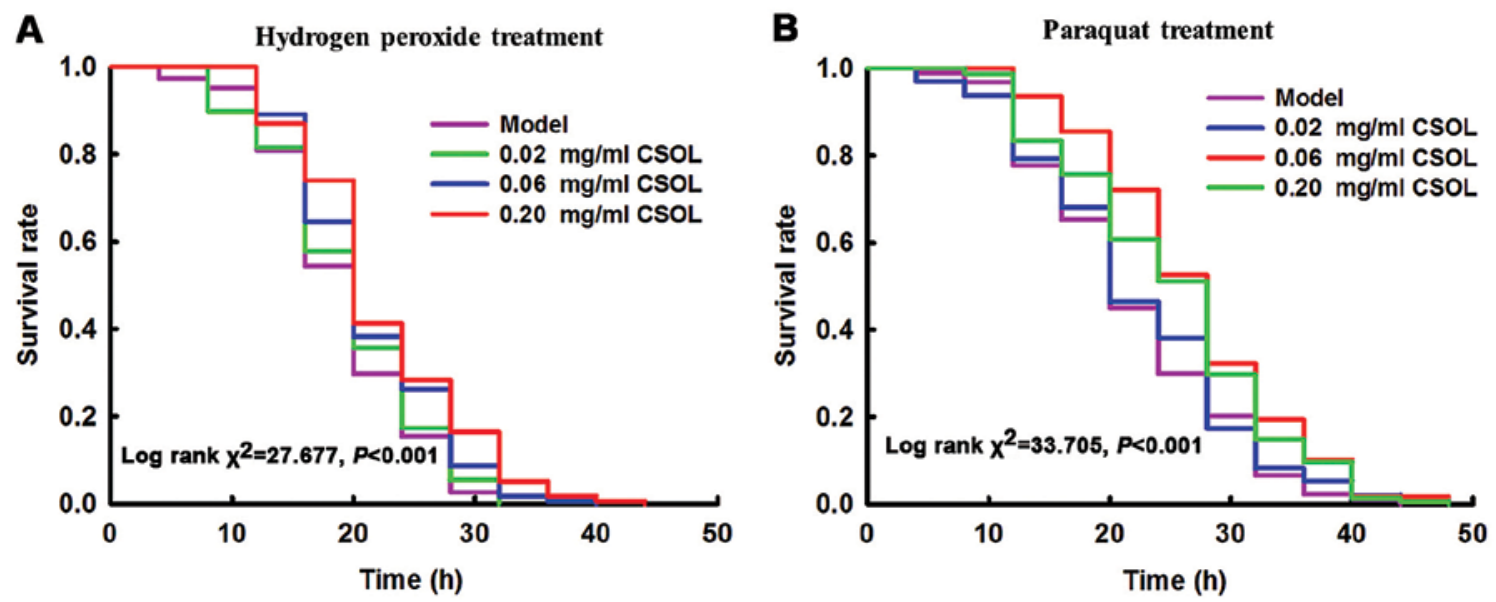

Figure 5. Cordyceps sinensis oral liquid (CSOL) inhibits damage induced by acute oxidative stress in fruit flies. Fruit flies (eclosion within 8 h) were treated with culture medium containing CSOL at concentrations of $0.02,0.06$ and $0.20 \mathrm{mg} / \mathrm{ml}$. Fruit flies in the model group were fed with basal culture medium. On day 25 , after starving for $2 \mathrm{~h}$, fruit flies were transferred to new vials containing a filter paper saturated with $1 \mathrm{ml}$ of $30 \%$ hydrogen peroxide $\left(\mathrm{H}_{2} \mathrm{O}_{2}\right)$, or with $1 \mathrm{ml}$ of $20 \mathrm{mM}$ paraquat diluted by $6 \%$ glucose solution, respectively. The number of dead flies were recorded every $4 \mathrm{~h}$ until all flies died. Data were analyzed by log-rank test. (A) CSOL inhibited damage induced by $\mathrm{H}_{2} \mathrm{O}_{2}$. Log-rank $\chi^{2}=27.677, \mathrm{P}<0.001, \mathrm{n}=200$. (B) CSOL inhibited damage induced by paraquat. Log-rank $\chi^{2}=33.705$, $\mathrm{P}<0.001, \mathrm{n}=200$.

in the fruit fly model group decreased significantly compared to the control group. The content of LF (Fig. 6E) increased significantly in the model group compared with the control group. Consistent with the results of our other experiments (described above), treatment with CSOL inhibited the decrease in SOD1 (Fig. 6B) and CAT (Fig. 6D) activity induced by D-galactose, and inhibited LF accumulation (Fig. 6E). CSOL had no significant effect on the activity of SOD2 (Fig. 6C).

\section{Discussion}

A suitable animal model is required for investigation of the anti-aging effects of CSOL. In the present study, we focused on the fruit fly $D$. melanogaster. It has been widely used as a model of aging for basic and applied research due to its advan- tages over mammalian models, such as high genus purity, strong reproductive capacity and a short lifespan (25). For these reasons, D. melanogaster was selected for use as a model organism in the study of the physiological and pathological processes affecting lifespan.

Our findings demonstrated that treatment with CSOL significantly prolonged the lifespan of the fruit flies. A previous study indicated that Cordyceps militaris extract possesses ROS scavenging activity which protects against premature senescence induced by oxidative stress (26). However, to the best of our knowledge, the present study used a model of physiological aging for the first time. This suggests that CSOL may have the potential to be exploited for anti-aging applications.

This prompted us to elucidate the mechanisms responsible for the lifespan-prolonging effects of CSOL. Oxidative stress 
Table III. Lifespan parameters in pathologically-aged fruit flies (exposed to D-galactose) following lifelong treatment with CSOL.

\begin{tabular}{|c|c|c|c|c|c|}
\hline $\begin{array}{l}\text { Group } \\
(\mathrm{mg} / \mathrm{ml})\end{array}$ & $\begin{array}{c}\text { Median lifespan } \\
\text { (day) }\end{array}$ & $\begin{array}{c}\text { Mean lifespan } \\
\text { (day) }\end{array}$ & $\begin{array}{c}\text { Change from } \\
\text { model }(\%)\end{array}$ & Log-rank (vs. model) & $\begin{array}{c}\text { Maximum lifespan } \\
\text { (day) }\end{array}$ \\
\hline Control & $55.0 \pm 1.7$ & $52.5 \pm 1.1$ & 25 & $\chi^{2}=74.033, P<0.001$ & $72.2 \pm 5.7^{\mathrm{a}}$ \\
\hline Model & $44.0 \pm 1.4$ & $42.1 \pm 0.8$ & - & - & $57.7 \pm 2.6$ \\
\hline 0.02 & $44.0 \pm 1.8$ & $44.7 \pm 1.0$ & 6 & $\chi^{2}=13.968, P<0.001$ & $64.4 \pm 3.4^{\mathrm{a}}$ \\
\hline 0.06 & $50.0 \pm 1.8$ & $45.6 \pm 1.0$ & 8 & $\chi^{2}=23.662, P<0.001$ & $65.2 \pm 4.5^{\mathrm{a}}$ \\
\hline 0.20 & $50.0 \pm 2.5$ & $47.2 \pm 1.2$ & 12 & $\chi^{2}=34.615, \mathrm{P}<0.001$ & $67.0 \pm 2.3^{\mathrm{a}}$ \\
\hline
\end{tabular}

Maximum lifespan was calculated as the average lifespan of the longest surviving $10 \%$ of fruit flies in each group $\left(\mathrm{n}=200,{ }^{\mathrm{a}} \mathrm{P}<0.001 \mathrm{vs}\right.$. the model group). CSOL, Cordyceps sinensis oral liquid.

A

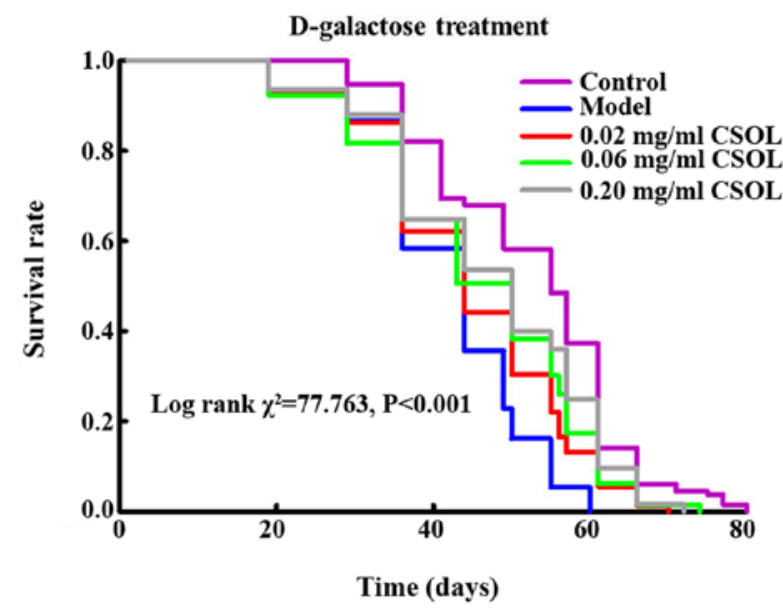

B

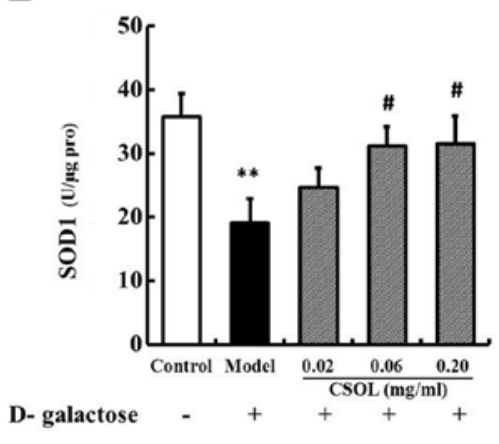

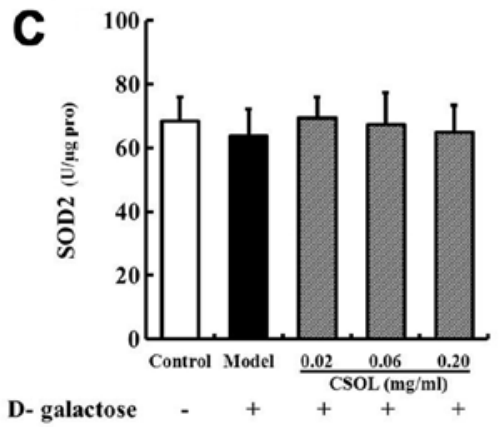
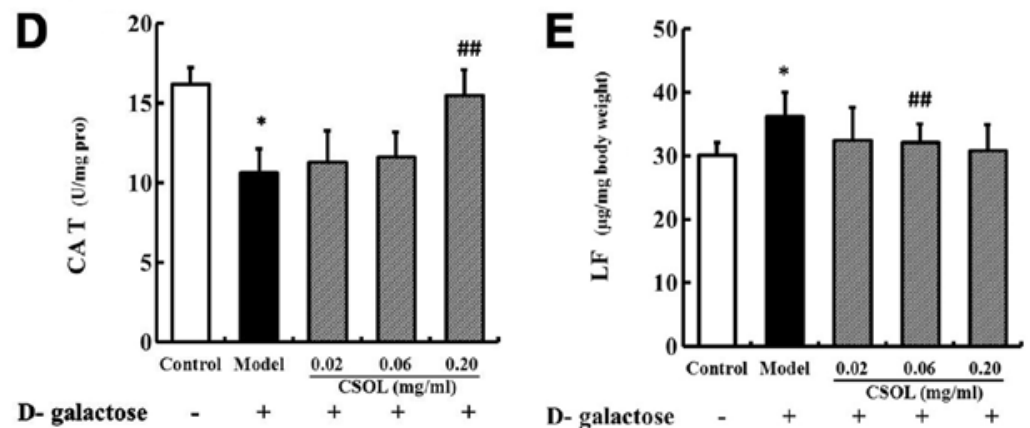

Figure 6. Cordyceps sinensis oral liquid (CSOL) inhibits pathological aging induced by D-galactose in fruit flies. Fruit flies (eclosion within 8 h) were treated with culture medium containing CSOL at concentrations of $0.02,0.06$ and $0.20 \mathrm{mg} / \mathrm{ml}$. Fruit flies in the control group and the model group were fed with basal culture medium. (A) CSOL treatment inhibited D-galactose-induced aging of fruit flies. Data were analyzed by log-rank test. Log-rank $\chi^{2}=77.763$, $\mathrm{P}<0.001$, $\mathrm{n}=200$. (B) CSOL inhibited the decreasing activity of superoxide dismutase (SOD)1 induced by D-galactose. (C) CSOL had no effect on the activity of SOD2. (D) CSOL inhibited the decreasing activity of catalase (CAT) induced by D-galactose. (E) CSOL inhibited lipofuscin (LF) accumulation induced by D-galactose. (B, C , D and E) Fruit flies were collected on day 25 for the detection of the activity of antioxidant enzymes. Data are presented as the means \pm SD. Data were analyzed by ANOVA followed by Dunnett's t-test. $\mathrm{n}=6,{ }^{*} \mathrm{P}<0.05,{ }^{* *} \mathrm{P}<0.01$ vs. the control group; ${ }^{\#} \mathrm{P}<0.05,{ }^{\# \#} \mathrm{P}<0.01$ vs. the model group.

has been implicated in aging and degenerative diseases (27). It is the result of increased ROS production (28), and causes progressive structural and functional alterations of cellular organelles. Antioxidants inhibit the process of aging (29). The continuous generation of ROS is also closely related to organism aging (30). There are two main assumptions of the free radical hypothesis of aging. Firstly, there are antioxidant defenses; organisms endogenously produce a group of antioxidant enzymes including SOD and CAT, which serve as the first line of defense against ROS. Secondly, a fraction of ROS escape elimination and are able to inflict molecular damage that accumulates with age, thereby causing the functional attrition associated with aging $(31,32)$.

Since oxidative stress is one of the mechanisms responsible for aging, we investigated the anti-oxidative stress effect of CSOL in fruit flies to further elucidate the mechanisms through which CSOL exerts a lifespan-prolonging effect. The upregulation of 
SOD1 and CAT activity, and the inhibition of LF accumulation suggested that the anti-aging effects of CSOL in fruit flies were associated, at least in part, with the upregulation of endogenous antioxidant enzymes, as well as the inhibition of LF accumulation. However, the unaltered mRNA levels of SOD1, SOD2 and CAT implied that CSOL had no effect at the transcriptional level of antioxidant genes in fruit flies. Thus, subsequent experiments mainly focused on the anti-oxidative stress effect of CSOL on the enzymes rather than on their transcriptional levels.

To confirm the anti-oxidative stress effect of CSOL, fruit flies were exposed to acute oxidative stress induced by either $\mathrm{H}_{2} \mathrm{O}_{2}$ or paraquat. The results, including the parameter of the mean survival time, further verified this effect.

To further examine the anti-oxidative stress effect of CSOL under pathological conditions, we selected a model of pathological aging induced by D-galactose in fruit flies. The results, including lifespan curves, and the mean and maximum lifespan parameters, confirmed the effects of CSOL. Additionally, the examination of SOD1 activity, CAT activity and the LF content in fruit flies, pathologically aged by D-galactose, further supported the theory that CSOL exerts an anti-aging effect through the anti-oxidative stress pathway under pathological conditions.

Taking into account these results, the anti-aging effect of CSOL, exerted through the anti-oxidative stress pathway, has been confirmed in both under physiological and pathological conditions in fruit flies.

In conclusion, our data demonstrated that CSOL prolonged the lifespan of fruit flies through an anti-oxidative stress pathway which involved the upregulation of the activity of SOD1 and CAT activity and the inhibition of LF accumulation. If the association between CSOL and anti-aging could be confirmed and the detailed mechanisms could be further clarified in future studies, it may provide a novel strategy for slowing the human aging process.

\section{Acknowledgements}

This study was supported by grants from the Natural Science Foundation of Shanghai City of China (no. 12ZR1444600 to Y.Z.) and the National Natural Science Foundation of China (no. 81402922 to Y.Z.).

\section{References}

1. Fontana L, Kennedy BK, Longo VD, Seals D and Melov S: Medical research: treat ageing. Nature 511: 405-407, 2014.

2. Hung WW, Ross JS, Boockvar KS and Siu AL: Recent trends in chronic disease, impairment and disability among older adults in the United States. BMC Geriatr 11: 47, 2011.

3. Dröge W: Oxidative stress and ageing: is ageing a cysteine deficiency syndrome? Philos Trans R Soc Lond B Biol Sci 360: 2355-2372, 2005.

4. Pham-Huy LA, He $\mathrm{H}$ and Pham-Huy C: Free radicals, antioxidants in disease and health. Int J Biomed Sci 4: 89-96, 2008.

5. López-Otín C, Blasco MA, Partridge L, Serrano M and Kroemer G: The hallmarks of aging. Cell 153: 1194-1217, 2013.

6. Höhn A and Grune T: Lipofuscin: formation, effects and role of macroautophagy. Redox Biol 1: 140-144, 2013.

7. Pincus Z, Smith-Vikos T and Slack FJ: MicroRNA predictors of longevity in Caenorhabditis elegans. PLoS Genet 7: e1002306, 2011.

8. Xiao JH, Xiao DM, Sun ZH, Xiao Y and Zhong IJ: Antioxidative potential of polysaccharide fractions produced from traditional Chinese medicinal macrofungus Cordyceps jiangxiensis in vitro. Afr J Biotechnol 10: 6607-6615, 2011.
9. Xiao JH, Xiao DM, Sun ZH, Xiong Q, Liang ZQ and Zhong JJ: Chemical compositions and antimicrobial property of three edible and medicinal Cordyceps species. J Food Agric Environ 7: 91-100, 2009.

10. Panda AK and Swain KC: Traditional uses and medicinal potential of Cordyceps sinensis of Sikkim. J Ayurveda Integr Med 2: 9-13, 2011.

11. Yamaguchi Y, Kagota S, Nakamura K, Shinozuka K and Kunitomo M: Antioxidant activity of the extracts from fruiting bodies of cultured Cordyceps sinensis. Phytother Res 14: 647-649, 2000.

12. Zhou X, Gong Z, Su Y, Lin J and Tang K: Cordyceps fungi: Natural products, pharmacological functions and developmental products. J Pharm Pharmacol 61: 279-291, 2009.

13. Li SP, Yang FQ and Tsim KW: Quality control of Cordyceps sinensis, a valued traditional Chinese medicine. J Pharm Biomed Anal 41: 1571-1584, 2006.

14. Ji DB, Ye J, Li CL, Wang YH, Zhao J and Cai SQ: Antiaging effect of Cordyceps sinensis extract. Phytother Res 23: 116-122, 2009.

15. Xiao JH, Xiao DM, Chen DX, Xiao Y, Liang ZQ and Zhong JJ: Polysaccharides from the medicinal mushroom Cordyceps taii show antioxidant and immunoenhancing activities in a D-galactose-induced aging mouse model. Evid Based Complement Alternat Med 2012: 273435, 2012.

16. Sun YH: Naval Medical Research Institute manufactured the 'immunomodulatory mixtures'. Navy Med 2: 11, 1993.

17. Wu JR, Chen DM, Sun YH, Ding JM and Zhu ZY: Study of Cordyceps capsules therapy on chronic obstructive lung disease. Chinese Patent Med Study (8): 26-27, 1986.

18. Falfushynska HI, Gnatyshyna LL, Osadchuk OY, Farkas A, Vehovszky A, Carpenter DO, Gyori J and Stoliar OB: Diversity of the molecular responses to separate wastewater effluents in freshwater mussels. Comp Biochem Physiol C Toxicol Pharmacol 164: 51-58, 2014

19. Zou YX, Zhang XH, Su FY and Liu X: Importance of riboflavin kinase in the pathogenesis of stroke. CNS Neurosci Ther 18: 834-840, 2012.

20. Lee KW and Lee HJ: Biphasic effects of dietary antioxidants on oxidative stress-mediated carcinogenesis. Mech Ageing Dev 127: 424-431, 2006

21. Goncalves RL, Rothschild DE, Quinlan CL, Scott GK, Benz CC and Brand MD: Sources of superoxide $/ \mathrm{H}_{2} \mathrm{O}_{2}$ during mitochondrial proline oxidation. Redox Biol 2: 901-909, 2014.

22. Dagda RK, Das Banerjee T and Janda E: How Parkinsonian toxins dysregulate the autophagy machinery. Int J Mol Sci 14: 22163-22189, 2013

23. Lei M, Hua X, Xiao M, Ding J, Han Q and Hu G: Impairments of astrocytes are involved in the D-galactose-induced brain aging. Biochem Biophys Res Commun 369: 1082-1087, 2008.

24. Lu J, Zheng YL, Luo L, Wu DM, Sun DX and Feng YJ: Quercetin reverses D-galactose induced neurotoxicity in mouse brain. Behav Brain Res 171: 251-260, 2006.

25. Kim SI, Jung JW, Ahn YJ, Restifob LL and Kwon HW: Drosophila as a model system for studying lifespan and neuroprotective activities of plant-derived compounds. J Asia Pac Entomol 14: 509-517, 2011.

26. Park JM, Lee JS, Lee KR, Ha SJ and Hong EK: Cordyceps militaris extract protects human dermal fibroblasts against oxidative stress-induced apoptosis and premature senescence. Nutrients 6: 3711-3726, 2014.

27. Ma Q: Advances in mechanisms of anti-oxidation. Discov Med 17: 121-130, 2014.

28. Apel K and Hirt H: Reactive oxygen species: Metabolism, oxidative stress, and signal transduction. Annu Rev Plant Biol 55: 373-399, 2004

29. Sadowska-Bartosz I and Bartosz G: Effect of antioxidants supplementation on aging and longevity. Biomed Res Int 2014: 404680, 2014.

30. Di DF, Barone E, Perluiqi M and Butterfield DA: Strategy to reduce free radical species in Alzheimer's disease: An update of selected antioxidants. Expert Rev Neurother 22: 1-22, 2014.

31. Hou S: Introduction to Aging: A positive, interdisciplinary approach. Health Promot Pract 16: 12-14, 2014.

32. Zarrouk A, Vejux A, Mackrill J, O'Callaghan Y, Hammami M, O'Brien $\mathrm{N}$ and Lizard G: Involvement of oxysterols in age-related diseases and ageing processes. Ageing Res Rev 18: 148-162, 2014. 